\title{
Effects of the combination of bone morphogenetic protein-2 and nano-hydroxyapatite on the osseointegration of dental implants
}

\author{
KangMi Pang ${ }^{1}$, Young-Kwon Seo ${ }^{2}$, Jong-Ho Lee ${ }^{3,4}$ \\ ${ }^{1}$ Department of Dentistry, Oral and Maxillofacial Surgery, Seoul National University Dental Hospital, \\ ${ }^{2}$ Department of Medical Biotechnology, College of Life Science and Biotechnology, Dongguk University, \\ ${ }^{3}$ Department of Oral and Maxillofacial Surgery, School of Dentistry, Seoul National University, \\ ${ }^{4}$ Dental Life Science Research Institute and Clinical Translational Research Center for Dental Science, \\ Seoul National University Dental Hospital, Seoul, Korea
}

\begin{abstract}
J Korean Assoc Oral Maxillofac Surg 2021;47:454-464)
Objectives: This study aimed to investigate the in vitro osteoinductivity of the combination of bone morphogenetic protein-2 (BMP-2) and nanohydroxyapatite (nHAp) and the in vivo effects of implants coated with nHAp/BMP-2.

Materials and Methods: To evaluate the in vitro efficacy of nHAp/BMP-2 on bone formation, bone marrow-derived mesenchymal stem cells (BMMSCs) were seeded onto titanium disks coated with collagen (Col), $\mathrm{Col} / \mathrm{nHAp}$, or Col/nHAp/BMP-2. Protein levels were determined by a biochemical assay and reverse transcriptase-polymerase chain reaction. Stem cell differentiation was analyzed by flow cytometry. For in vivo studies with mice, $\mathrm{Col}, \mathrm{Col} / \mathrm{nHAp}$, and Col/nHAp/BMP-2 were injected in subcutaneous pockets. Titanium implants or implants coated with Col/nHAp/BMP-2 were placed bilaterally on rabbit tibias and evaluated for 4 weeks.

Results: In the in vitro study, BM-MSCs on Col/nHAp/BMP-2 showed reduced levels of CD73, CD90, and CD105 and increased levels of glycosaminoglycan, osteopontin, and alkaline phosphatase activity. After 4 weeks, the Col/nHAp/BMP-2 implant showed greater bone formation than the control $(P=0.07)$, while no differences were observed in bone implant contact and removal torque.
\end{abstract}

Conclusion: These results suggest that a combination of BMP-2 and an nHAp carrier would activate osseointegration on dental implant surfaces.

Key words: Bone morphogenetic protein 2, Hydroxyapatite, Collagen, Dental implant, Osseointegration

[paper submitted 2021. 10. 15 / revised 2021. 10. 20 / accepted 2021. 10. 21]

\section{Introduction}

Studies have been directed towards improving the osseointegration of dental implants by modifying the implant surface ${ }^{1}$. Surface topography and chemical modification are two important elements that influence the bone/implant interface ${ }^{2}$. Biochemical modification refers to the immobilization of bioactive peptides or growth factors such as bone morphogenetic

\footnotetext{
Jong-Ho Lee

Department of Oral and Maxillofacial Surgery, Dental Life Science Research Institute and Clinical Translational Research Center for Dental Science, Seoul National University Dental Hospital, 101 Daehak-ro, Jongno-gu, Seoul 03080, Korea

TEL: +82-2-2072-2630

E-mail:leejongh@snu.ac.kr

ORCID: https://orcid.org/0000-0002-8843-545X
}

(c) This is an open-access article distributed under the terms of the Creative Commons Attribution Non-Commercial License (http://creativecommons.org/ licenses/by-nc/4.0/), which permits unrestricted non-commercial use, distribution, and reproduction in any medium, provided the original work is properly cited.

Copyright (C) 2021 The Korean Association of Oral and Maxillofacial Surgeons. protein $(\mathrm{BMP})^{3}$.

BMP-2, a member of the transforming growth factor beta superfamily, binds to the receptors of mesenchymal stem cells, osteoblasts, and osteoclasts, and induce cell chemotaxis, proliferation, and differentiation. Dental implants placed in bone whose formation is induced by BMP-2 have been reported to exhibit successful osseointegration and respond well to functional loading, as do dental implants placed in native alveolar bone ${ }^{4,5}$. High doses of BMP-2 are known to directly induce bone formation ${ }^{6}$. While BMP-2 has yielded promising results regarding osteogenesis, high dosage may produce detrimental side effects, including heterotopic bone formation, soft-tissue swelling, seroma formation, osteolysis, radiculopathy, and malignancy. Therefore, to achieve bone induction, a sustained-release carrier is needed for BMP-2 due to its short biological half-life and rapid diffusion when implanted in vivo ${ }^{6-14}$.

Many materials have been tested as a delivery system for 
BMP, including synthesized polymers, collagen, tricalcium phosphate, polyethylene glycol hydrogels, and hydroxyapatite (HAp) $)^{6,15}$. HAp has been applied to clinically approved bone substitutes due to its high osteoconductivity and maintenance of a constant shape. It also exhibits a high affinity for BMP-2, evenly distributes BMP-2 under pressure, and causes minimal foreign body reactions, and is therefore considered useful as a BMP carrier ${ }^{16}$. Lee et al. ${ }^{6}$ reported excellent results when BMP-2 was combined with HAp as bone-grafting material in a rabbit spinal fusion model. Furthermore, recent studies have produced improved results for implant surfaces coated with BMP-2. To our knowledge, no studies have been performed on the efficacy and effects of combining BMP-2 with nano-hydroxyapatite (nHAp) on implant surfaces. We hypothesized that a surface coating combining BMP-2 with nHAp would improve osseointegration; therefore, we have evaluated the osteoinductivity of BMP-2 combined with nHAp on dental implant surfaces in vitro and in vivo.

\section{Materials and Methods}

\section{Preparation of titanium disks}

Grade-4 titanium foils $(5 \mathrm{~cm} \times 5 \mathrm{~cm})$ were used. nHAp solution was purchased from Sigma-Aldrich Chemical (St. Louis, MO, USA). Human BMP-2 was donated by Daewoong Pharmaceutical (Seoul, Korea). The surface of each titanium disk was etched by sonification of the disks in Kroll's reagent (4.0\% $\mathrm{HF}, 7.2 \% \mathrm{HNO}_{3}$, and $88.8 \%$ water) for 10 minutes. The disks were then washed ultrasonically for 10 minutes each in acetone and triple-distilled water and placed in $40 \%$ $\mathrm{HNO}_{3}$ for 40 minutes in order to passivate the surface.

\section{Preparation of collagen/nHAp/BMP-2 composite solution and coating}

The composite solution consisted of collagen (Col), nHAp, and BMP-2. A type I atelocollagen powder (Koken, Osaka, Japan) was dissolved at a concentration of $5 \mathrm{mg} / \mathrm{mL}$, and chondroitin-6-sulfate, nHAp, and BMP-2 were dissolved at concentrations of $0.25 \mathrm{mg} / \mathrm{mL}, 0.03 \mathrm{mg} / \mathrm{mL}$, and $100 \mu \mathrm{g} / \mathrm{mL}$, respectively. The composite solution $(500 \mu \mathrm{L})$ was coated onto the titanium foil $(5 \mathrm{~cm} \times 5 \mathrm{~cm})$, and the foil was dried for 24 hours at $4^{\circ} \mathrm{C}$. For implants attached to the rabbit tibias, 20 $\mu \mathrm{L}$ of the composite solution was coated onto each implant, and the implant was dried for 24 hours at $4^{\circ} \mathrm{C}$. The surfaces of the various titanium disks were characterized by static contact angle and atomic force microscopy (AFM).

\section{AFM}

To scan the surface, Nanomen II AFM (Veeco, Plainview, NY, USA) was used. Scans and readings were taken by tapping mode AFM at random sites ( $1 \mathrm{~mm}^{2}$ each) for 3 disks using a 5-mm scan head. A cantilever with an attached tip was oscillated at its resonant frequency to scan across the sample surface. Typical amplitudes were 20-100 nm, which could vary when the tip scanned over bumps or depressions on a surface. The van der Waals forces were $200 \mathrm{pN}$ or less. The surface roughness (root mean square [RMS]) was calculated using the Nanomen II software.

\section{Contact angle}

To measure the contact angle, a contact angle analyzer (DSA100; Krüss, Nürnberg, Germany) was used with $2 \mu \mathrm{m}$ deionized water. The contact angle of the water droplet was measured after 10 seconds on both sides of the water droplet, and 5 samples per group were evaluated.

\section{Release of BMP-2 from the disk}

Release of BMP-2 from a bare titanium surface, Col, Col/ BMP-2, or Col/nHAp/BMP-2 was determined by first incubating the disks with phosphate-buffered saline (PBS) at $37^{\circ} \mathrm{C}$ in sealed tubes blocked with bovine serum albumin and then recovering the released media at 1, 2, 3, 5, 7, and 9 days, followed by replacement with new PBS. BMP-2 was measured indirectly in the release media by using the human BMP-2 super X-ELISA kit obtained from Antigenix America (Huntington Station, NY, USA). Each group included 3 samples for loading and release.

\section{Bone marrow-derived mesenchymal stem cell culture}

Human bone marrow-derived mesenchymal stem cells (BMMSCs) (passage 2) were purchased from Lonza (Portsmouth, $\mathrm{NH}$, USA). The mononuclear cell fraction was isolated by Ficoll $(0.77 \mathrm{~g} / \mathrm{mL})$ density gradient centrifugation. BM-MSCs $\left(2 \times 10^{5}\right.$ cells) were seeded onto the $5 \mathrm{~cm} \times 5 \mathrm{~cm}$ titanium disks. The osteogenic medium consisted of DMEM containing $10 \%$ fetal bovine serum, $10 \mathrm{mM} \beta$-glycerophosphate, $50 \mu \mathrm{M}$ Asc2-P, and $10^{-7} \mathrm{M}$ dexamethasone (all from Sigma-Aldrich), and the medium was changed every 3 to 4 days. 


\section{Flow cytometry (FACS) after differentiation}

Antibodies against human antigens CD90 and CD73 were purchased from BD Sciences (San Jose, CA, USA), and the antibody against CD105 was purchased from Ancell (Bayport, MN, USA). The fluorescence intensity of the cells was evaluated by a flow cytometer (FACScan; BD Sciences), and the data were analyzed using the CELLQUEST software (BD Sciences).

\section{Biochemical assays for alkaline phosphatase (ALP), glycosaminoglycan (GAG), and osteopontin (OPN) production}

ALP activity was measured from the cell supernatant by using SensoLyte pNPP Alkaline Phosphatase Assay Kit "Colorimetric" (AnaSpec, Fremont, CA, USA). The total intracellular sulfated GAG content was measured using a BlyscanSulfated Glycosaminoglycans Assay Kit (Bioassay, Hayward, CA, USA). The concentration of OPN protein in the culture medium was measured using a Quantikine Human Osteopontin Immunoassay kit (R\&D Systems, Minneapolis, MN, USA). The samples were dissolved in dissociation reagent, and the absorbance was measured at $656 \mathrm{~nm}$ using a microplate reader (VERSAmax Tunable Microplate Reader; Molecular Devices, Sunnyvale, CA, USA).

\section{Reverse transcription-polymerase chain reaction (RT-PCR) for BM-MSCs}

RT-PCR analysis was performed to compare the expression of bone-inducing markers such as type I collagen, type
III collagen, osteonectin, OPN, BMP-2, osteoprotegerin, and GAPDH (internal control). Total cellular RNA was isolated by Trizol reagent (Invitrogen, Carlsbad, CA, USA). cDNA was synthesized by reverse transcription. The primers used for RT-PCR were purchased from Bioneer (Daejeon, Korea), and their sequences, reaction conditions, and product size (bp) are summarized in Table 1. The PCR products were then analyzed on $2 \%$ agarose gels and visualized by SYBR safe DNA gel staining (Invitrogen). The Image J software (National Institutes of Health, Bethesda, MD, USA) was used for quantitative analysis of RT-PCR amplicons on digitized gel images.

\section{Mouse subcutaneous injection}

The experiments were approved by the Animal Care and Use Committee, Seoul National University (SNU-1001228). For all experiments, the animals were housed and handled in strict accordance with the animal care guidelines of the Institute of Laboratory Animal Resources, Seoul National University. Col, Col/nHAp, or Col/nHAp/BMP-2 was added to $20 \mu \mathrm{L}$ of mixing solution and injected into the subcutaneous pockets of nude mice.

\section{Immunohistochemical staining}

Mice were sacrificed and examined 4 weeks after injection of a composite solution. H\&E stain, Masson's trichrome (MT) stain, and immunochemistry were performed to assess bone formation and angiogenesis from the injected composite solution. Anti-osteonectin (1:500 dilution, AB 1858; Chemicon, Carlsbad, CA, USA) was used for osteonectin staining and anti-CD31 monoclonal antibody (Dako, Carpinteria, CA,

Table 1. Primer sequences, reaction conditions, and product size for RT-PCR

\begin{tabular}{|c|c|c|c|c|}
\hline Gene & Sequence & Product size (bp) & Annealing $\left({ }^{\circ} \mathrm{C}\right)$ & Cycles \\
\hline GAPDH & F: ACC ACA GTC CAT GCC ATC AC & 450 & 55 & 25 \\
\hline & R: TTC ACC ACC CTG TTG CTG TA & & & \\
\hline Collagen1 & $\begin{array}{l}\text { F: GAA AAC ATC CCA GCC AAG AA } \\
\text { R: CAG GTT GCC AGT CTC CTC AT }\end{array}$ & 270 & 57 & 23 \\
\hline Collagen3 & $\begin{array}{l}\text { F: CAG GTG AAC GTG GAG CTG C } \\
\text { R: TGC CAC ACG TGT TTC CGT GG }\end{array}$ & $\begin{array}{l}661 \\
849\end{array}$ & 57 & 23 \\
\hline Osteonectin & $\begin{array}{l}\text { F: CCA GAA CCA CCA CTG CAA AC } \\
\text { R: GGC AGG AAG AGT CGA AGG TC }\end{array}$ & 161 & 57 & 23 \\
\hline Osteopontin & $\begin{array}{l}\text { F: TCG CAG ACC TGA CAT CCA GT } \\
\text { R: TCG GAA TGC TCA TTG CTC TC }\end{array}$ & 267 & 57 & 32 \\
\hline$B M P-2$ & $\begin{array}{l}\text { F: GTC CAG CTG TAA GAG ACA CC } \\
\text { R: GTA CTA GCG ACA CCC ACA AC }\end{array}$ & 316 & 54 & 31 \\
\hline
\end{tabular}

(RT-PCR: reverse transcription-polymerase chain reaction, GAPDH: glyceraldehyde 3-phosphate dehydrogenase, BMP-2: bone morphogenetic protein-2)

KangMi Pang et al: Effects of the combination of bone morphogenetic protein-2 and nano-hydroxyapatite on the osseointegration of dental implants. J Korean Assoc Oral Maxillofac Surg 2021 
USA) for CD31 staining, followed by standard immunohistochemical procedures. The images were examined under a light microscope (System Microscope CX 40-32PH; Olympus, Center Valley, PA, USA).

\section{Implant installation on the rabbit tibia and removal torque measurement}

Titanium implants treated with resorbable blasted media were used as the negative control while implants coated with $\mathrm{Col} / \mathrm{nHAp} / \mathrm{BMP}-2$ were used as the experimental group. The mean weight of the rabbits was $3.3 \pm 0.25 \mathrm{~kg}$ at the time of the operation. The diameter and length of the implant were 3.3 $\mathrm{mm}$ and $7 \mathrm{~mm}$, respectively. Two control implants were randomly placed in the right or left side tibia of each animal, and 2 experimental implants were placed in the contralateral side tibia. The animals were sacrificed after a follow-up period of 4 weeks. The implants on the proximal side were used for histomorphometric analysis, and those on the distal side were used for the removal torque test. Static torque was applied at a linearly increasing rate of $9.5 \mathrm{Ncm} / \mathrm{second}$. The strength and rate of osseointegration of the bone implant were evaluated by the peak removal torque values (RTQ, Ncm).

\section{Histological analysis}

The fixated specimens were dehydrated in a graded ethanol series by using a dehydration system with agitation and a vacuum and embedded in light-curing methacrylate (Technovit
7200 VCL; Kulzer, Wehrheim, Germany). The implants were cut in a mid-axial coronal-apical plane using the sawing-andgrinding technique (EXAKT Apparatebau, Norderstedt, Germany). The bone implant contact (BIC) ratio and new bone formation area inside the thread area of the upper 3 threads were measured.

\section{Statistical analysis}

Statistical analysis was performed using the PASW Statistics software (ver. 18.0; IBM, Armonk, NY, USA). Numerical data are presented as mean values with the standard deviations. The Mann-Whitney test was carried out to analyze the differences in parameters of the rabbit implant installation model.

\section{Results}

\section{Effect of $n H A p$ and BMP-2 on titanium surface roughness and chemistry}

$\mathrm{Col} / \mathrm{nHAp} / \mathrm{BMP}-2$ increased the surface roughness more than Col, Col/BMP-2, and the bare titanium surface.(Fig. 1) The RMS values of the negative control, Col, Col/BMP-2, and $\mathrm{Col} / \mathrm{nHAp} / \mathrm{BMP}-2$ were $357,535,539$, and $872 \mathrm{~nm}$, respectively.

The water contact angles on the bare titanium surface, $\mathrm{Col}, \mathrm{Col} / \mathrm{BMP}-2$, and $\mathrm{Col} / \mathrm{nHAp} / \mathrm{BMP}-2$ were $70.5^{\circ} \pm 8.2^{\circ}$, $42.2^{\circ} \pm 3.6^{\circ}, 41.4^{\circ} \pm 2.6^{\circ}$, and $58.4^{\circ} \pm 3.0^{\circ}$, respectively. (Fig.

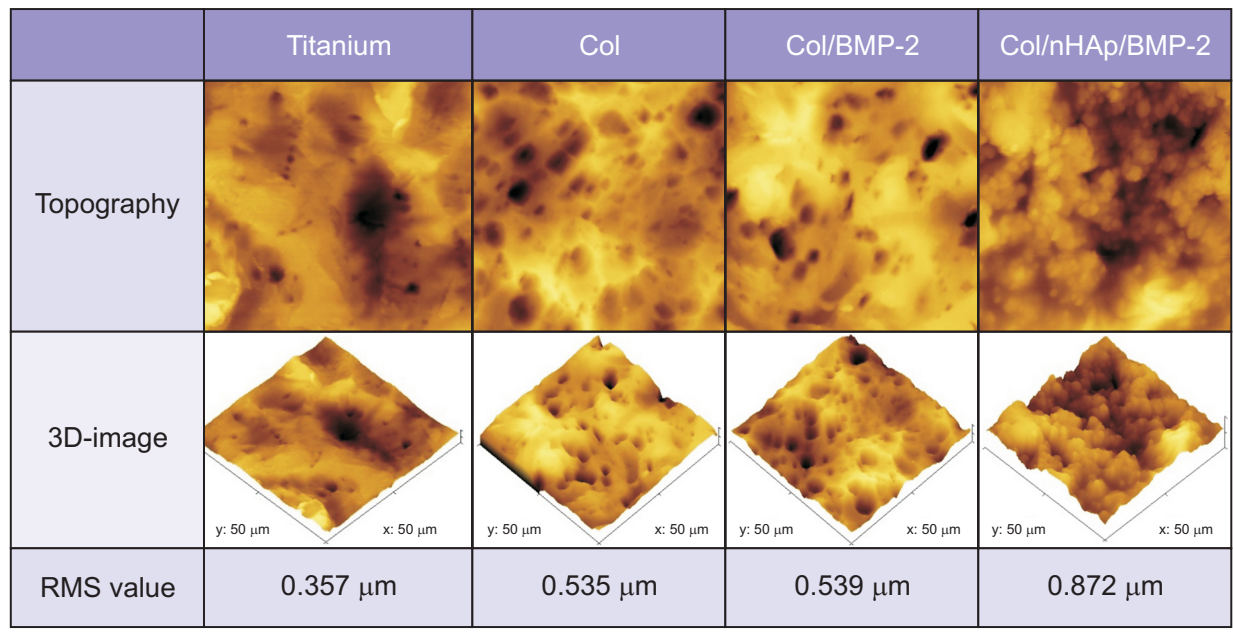

Fig. 1. Topographic and three-dimensional atomic force microscopic images of the coatings. Titanium: untreated titanium disk, Col: collagen-coated titanium surface, Col/BMP-2: collagen/bone morphogenetic protein-2 (BMP-2)-coated titanium surface, Col/nHAp/BMP-2: collagen/nano-hydroxyapatite/BMP-2-coated titanium surface. (RMS: root mean square)

KangMi Pang et al: Effects of the combination of bone morphogenetic protein-2 and nano-hydroxyapatite on the osseointegration of dental implants. J Korean Assoc Oral Maxillofac Surg 2021 

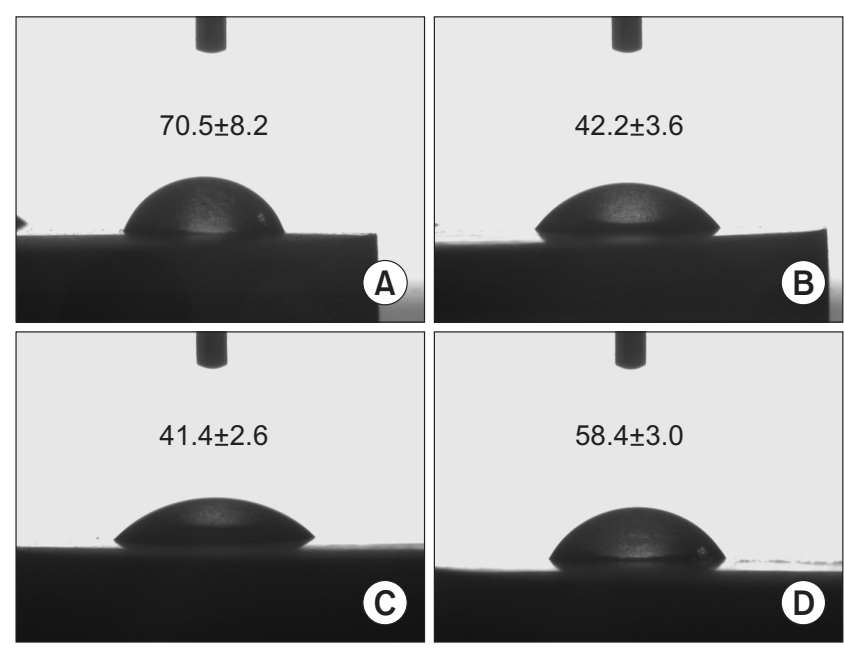

Fig. 2. Static water contact angle on titanium surface. A. Negative control (resorbable blasted media surface titanium). B. Collagencoated surface. C. Collagen/BMP-2-coated surface. D. Collagen/ nHAp/BMP-2-coated surface. (BMP-2: bone morphogenetic protein-2, nHAp: nano-hydroxyapatite)

KangMi Pang et al: Effects of the combination of bone morphogenetic protein-2 and nano-hydroxyapatite on the osseointegration of dental implants. J Korean Assoc Oral Maxillofac Surg 2021

2) These results indicated that the collagen-coated titanium surface increased hydrophilicity, while the addition of nHAp slightly reduced the hydrophilicity of the collagen-coated titanium surface.

\section{2. $\mathrm{BMP}-2$ release}

A solution containing $200 \mathrm{ng}$ of BMP-2 was coated onto the titanium surfaces. Surfaces treated with Col/nHAp/BMP2 exhibited a release of approximately 50\% after 24 hours incubation and approximately $75 \%$ after 48 hours incubation, while Col/BMP-2 exhibited a 70\% initial release within 24 hours, followed by an $88 \%$ release at 48 hours.(Fig. 3) Therefore, most of the coated BMP-2 was released within 5 days from Col/nHAp/BMP-2 and within 3 days from Col/BMP-2.

\section{FACS analysis after differentiation}

BM-MSCs were tested for the presence of mesenchymal stem cell markers, including the antigens CD73, CD90, and CD105, with flow cytometry.(Fig. 4. A) BM-MSCs exhibited high expression of these markers prior to differentiation. Expression was down-regulated following culturing on the titanium surface coated with Col/nHAp/BMP-2 after 2 weeks.

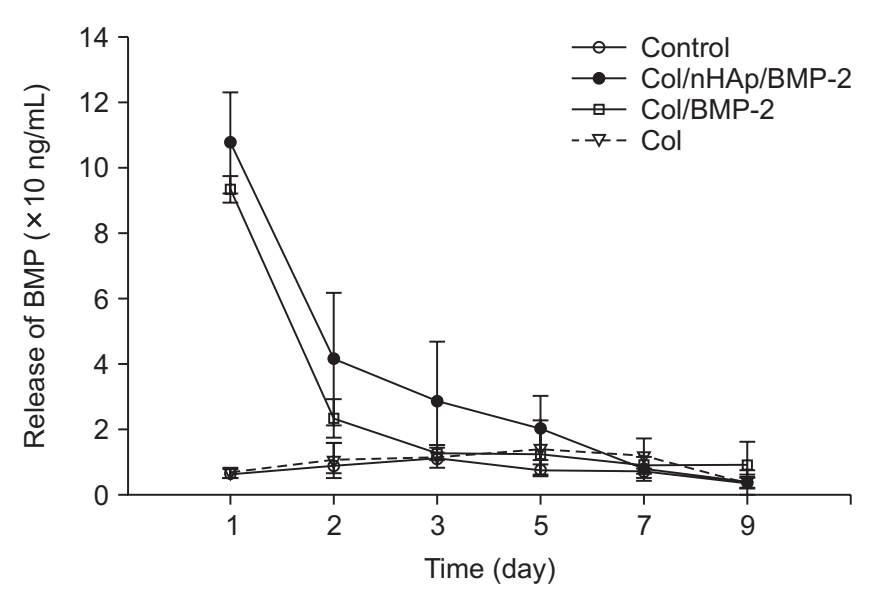

Fig. 3. In vitro release curve of bone morphogenetic protein-2 (BMP-2) from various surface-treated titanium disks. The results are shown as mean \pm standard deviation values $(n=3)$. An increase in BMP-2 release was observed within 24 hours. A sustained release in the Col/nHAp/BMP-2 group was observed between 1-5 days. (Col: collagen, $\mathrm{nHAp:} \mathrm{nano-hydroxyapatite)}$

KangMi Pang et al: Effects of the combination of bone morphogenetic protein-2 and nano-hydroxyapatite on the osseointegration of dental implants. J Korean Assoc Oral Maxillofac Surg 2021

\section{Biochemical assays for ALP, GAG, and OPN production}

The BM-MSCs were cultured for 14 days, and assays for ALP, GAG, and OPN production were performed to evaluate bone induction.(Fig. 4. B) In the cells grown on the $\mathrm{Col} /$ nHAp/BMP-2 surface, ALP activity and GAG and OPN levels were significantly higher than those in the cells grown with Col enrichment. Cells on the Col/nHAp/BMP-2 surface also exhibited higher ALP activity and GAG and OPN levels than those on the Col/nHAp surface. These findings indicated that BMP-2 with nHAp maintains its biological activity and induces bone development in BM-MSCs.

\section{RT-PCR}

As seen in Fig. 4. C, type I and III collagen levels significantly increased in BM-MSCs grown on Col/nHAp and Col/ nHAp/BMP-2, respectively. A similar expression pattern was observed for osteocalcin and osteoprotegerin in cells grown on Col/nHAp/BMP-2. Both the Col/nHAp and Col/nHAp/ BMP-2 groups had higher levels of OPN, osteonectin, and BMP-2 expression than the Col group.

\section{Mouse subcutaneous injections}

At the 4th week, no obvious bone formations were observed for any of the injected materials, either macroscopi- 
A
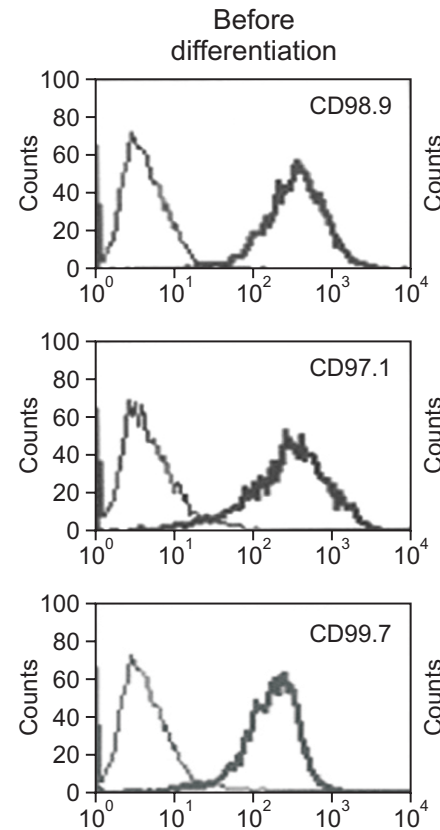

Col

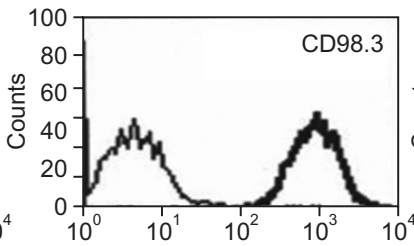

$\mathrm{Col} / \mathrm{nHAp}$
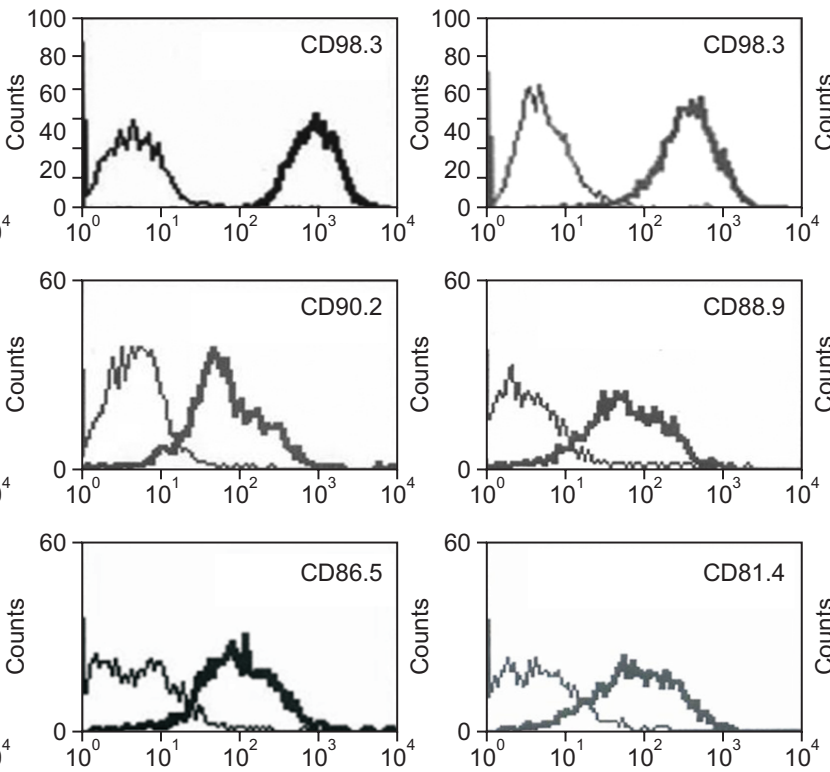

Col/nHAp/BMP-2
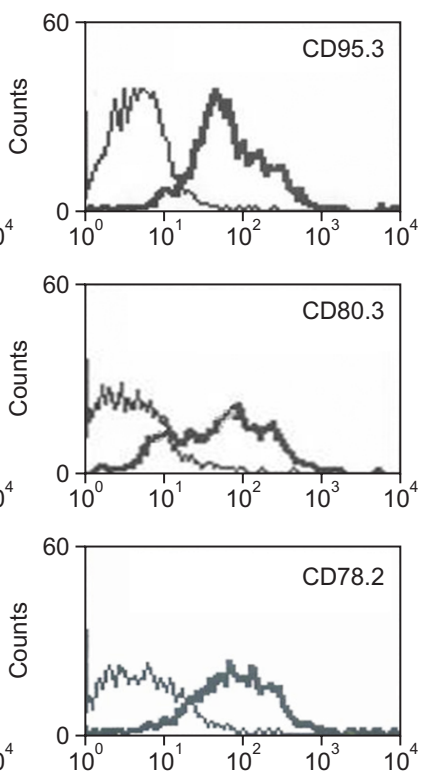

B
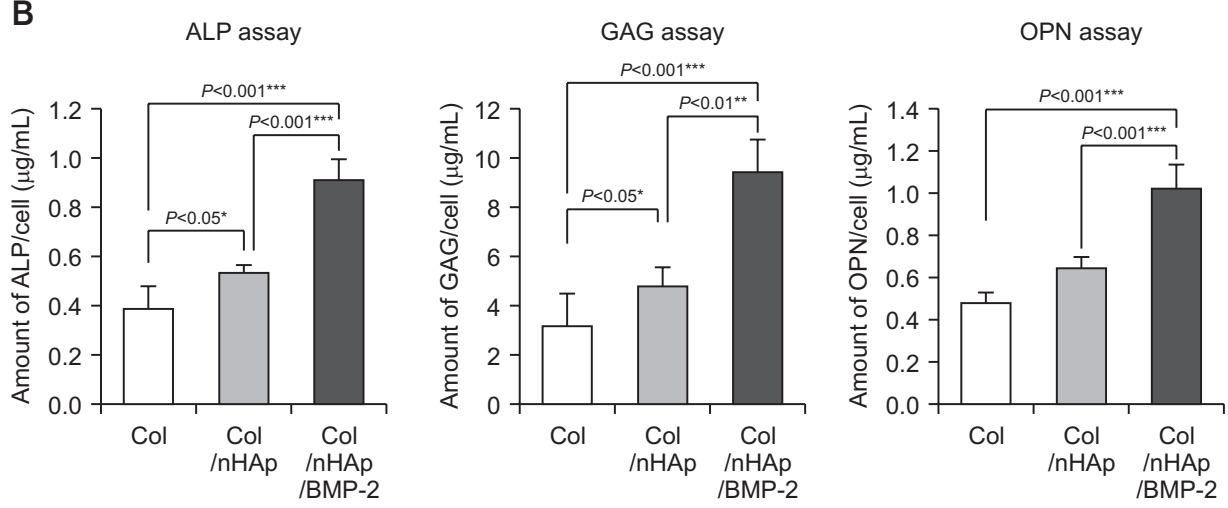

C

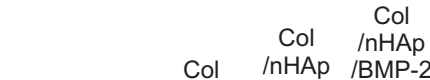

Fig. 4. Bone marrow-derived mesenchymal stem cell (BM-MSC) differentiation on differently treated titanium disks. A. Flow cytometry of typical CD markers present on BM-MSCs cultured on the Col-, Col/nHAp-, or Col/nHAp/BMP-2-coated titanium disks. CD73+, CD90+, and CD105+ cells were observed prior to differentiation. Reduction in mesenchymal CD73+, CD90+, and CD105+ cells was observed in the $\mathrm{Col} / \mathrm{nHAp} / \mathrm{BMP}-2$ group. B. Biochemical assays for alkaline phosphatase (ALP) activity and intracellular glycosaminoglycan (GAG) and osteopontin expression in BM-MSCs. Cells on Col/nHAp/BMP-2 exhibited the highest expression. C. mRNA expression of different osteoblastic markers in BM-MSC. The highest expression was observed for type III collagen (Col III), osteocalcin, and osteoprotegerin in cells grown on Col/nHAp/BMP-2. The levels of type I collagen (Col I), osteopontin, osteonectin, and BMP-2 expression were higher in BMMSCs on Col/nHAp and Col/nHAp/BMP-2 than in those with Col enrichment. (Col: collagen, nHAp: nano-hydroxyapatite, BMP-2: bone morphogenetic protein-2)

KangMi Pang et al: Effects of the combination of bone morphogenetic protein-2 and nano-hydroxyapatite on the osseointegration of dental implants. J Korean Assoc Oral Maxillofac Surg 2021

cally or microscopically (Fig. 5. A-I), although many cells had grown into both $\mathrm{Col} / \mathrm{nHAp}$ and col/nHAp/BMP-2. Additionally, the injected materials were partially degraded. Application of von Kossa staining revealed the absence of mineralization in the Col group (Fig. 5. J), with higher intensity observed for Col/nHAp than for Col/nHAp/BMP-2.(Fig. 5. K, 5. L) Differentiation of osteoblasts was confirmed by staining for osteonectin. Staining for osteonectin was limited in the case of Col enrichment, whereas the strongest staining was observed in Col/nHAp/BMP-2.(Fig. 5. M-O) For evaluating angiogenesis, tissues surrounding the injected composite solution were stained for CD31. A higher degree of CD31 staining and angiogenesis was observed in $\mathrm{Col} / \mathrm{nHAp} / \mathrm{BMP}-2$ than in Col/nHAp.(Fig. 5. P-R) 

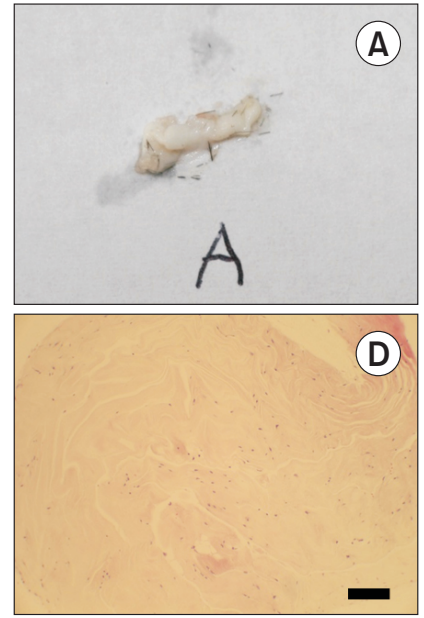

D)
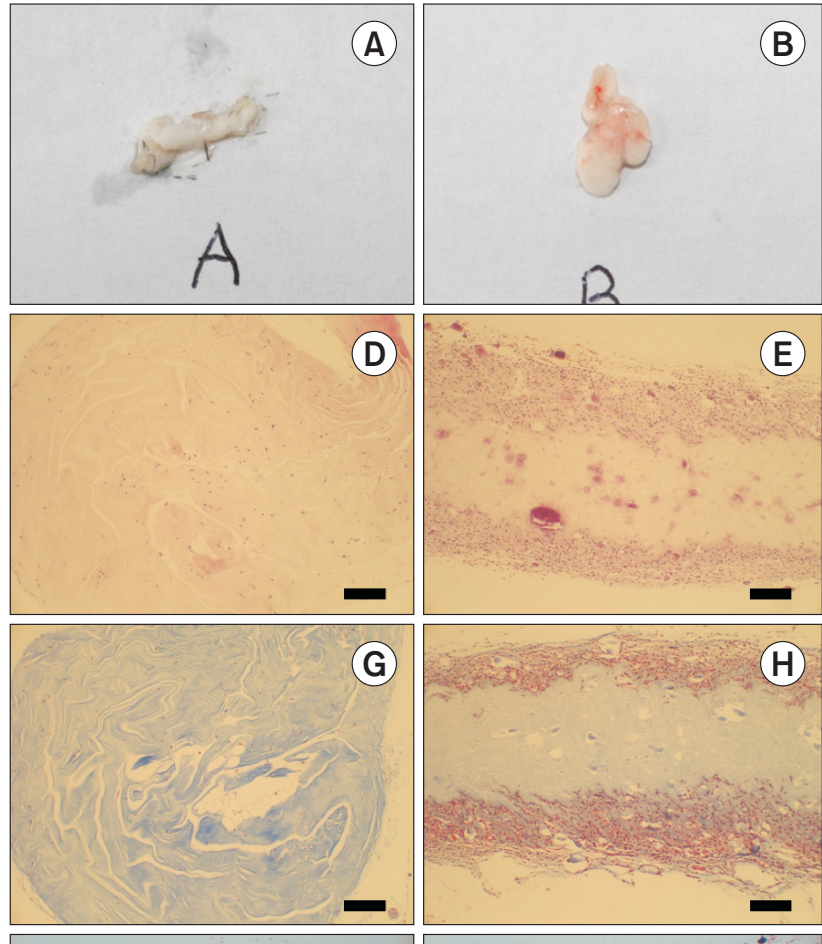

(J)
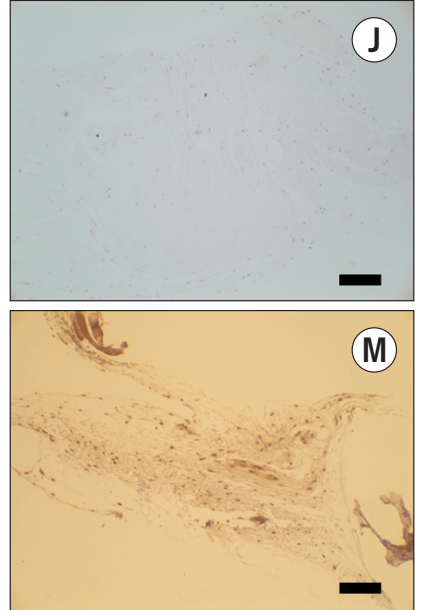

M)

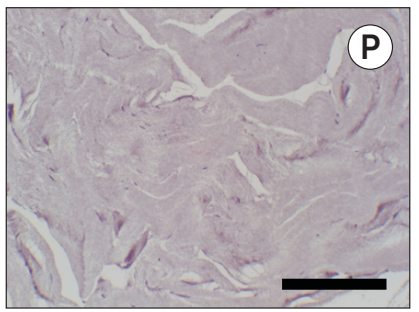

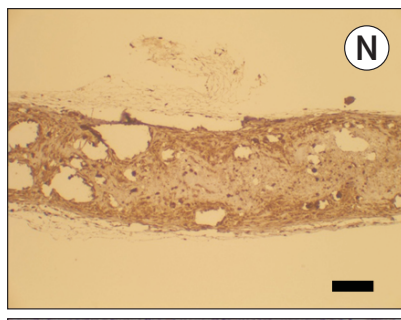
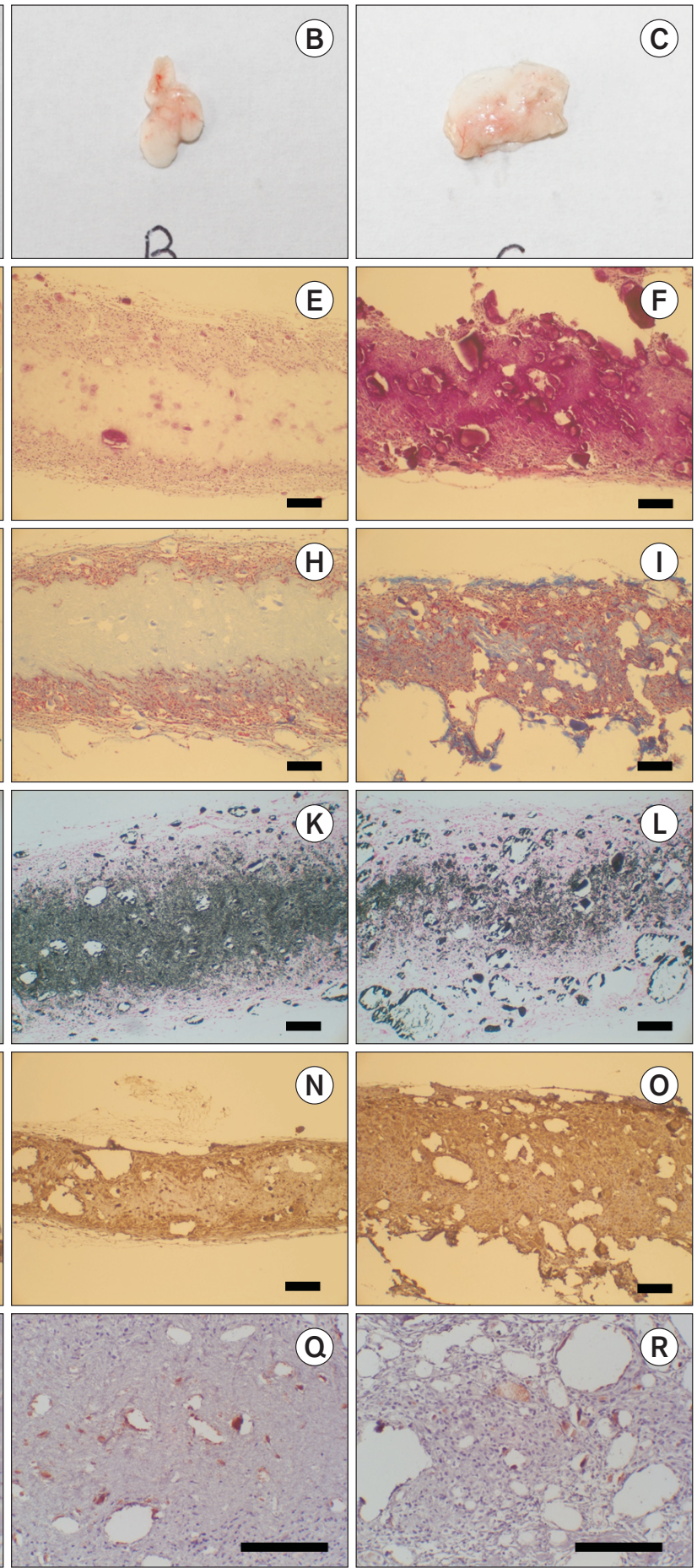

Fig. 5. Histological images of the mouse subcutaneous pocket injection model. Col (A, D, G, J, M, P), Col/nHAp (B, E, H, $\mathrm{K}, \mathrm{N}, \mathrm{Q}$ ), and $\mathrm{Col} / \mathrm{nHAp} / \mathrm{BMP}(\mathrm{C}, \mathrm{F}, \mathrm{I}, \mathrm{L}$, $\mathrm{O}, \mathrm{R})$. Macroscopic images (A-C), H\&E staining (D-F), Masson's trichrome (MT) staining $(G-I)$, von Kossa staining (J-L), osteonectin staining (M-O), and CD31 staining $(P-R)(D-O: \times 100, P-R: \times 200$, scale bars $=200 \mu \mathrm{m})$. (Col: collagen, nHAp: nano-hydroxyapatite, BMP-2: bone morphogenetic protein-2)

KangMi Pang et al: Effects of the combination of bone morphogenetic protein-2 and nano-hydroxyapatite on the osseointegration of dental implants. J Korean Assoc Oral Maxillofac Surg 2021

\section{Implant installation on the rabbit tibia}

No differences in stability were observed between the 2 types of implants. Results of the histomorphometric analysis for the 4-week specimens are summarized in Fig. 6. Following 4 weeks of healing, the Col/nHAp/BMP-2 implant demonstrated slightly more new bone area than the negative control $(P=0.07)$, whereas BIC and removal torque showed no significant differences, although the mean values were higher. The Col/nHAp/BMP-2 demonstrated a greater BIC $(33.46 \% \pm 6.91 \%)$ than the titanium implant $(31.36 \% \pm 3.22 \%)$, but the differences were not statistically significant. New bone area in the Col/nHAp/BMP-2 (73.69\% $\pm 5.88 \%)$ was slightly higher than that of the titanium implant surface $(62.24 \% \pm 12.52 \%)$. Similarly, Col/nHAp/BMP-2 had a greater removal torque $(26.63 \pm 4.41 \mathrm{Ncm})$ than the negative 
A

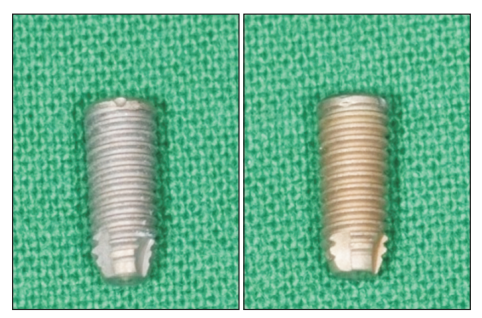

B

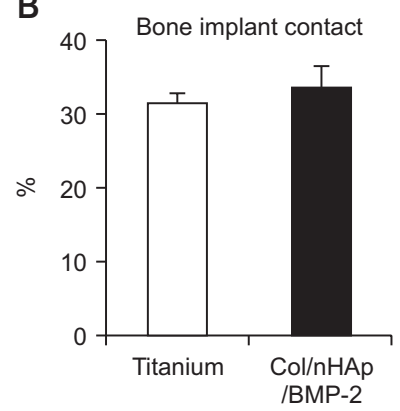

C

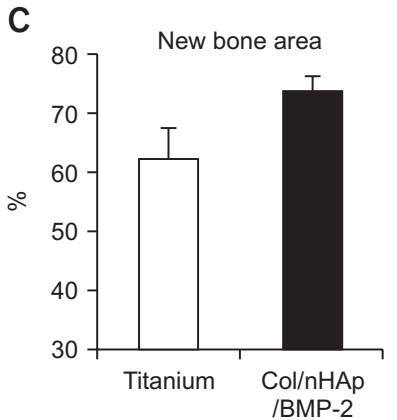

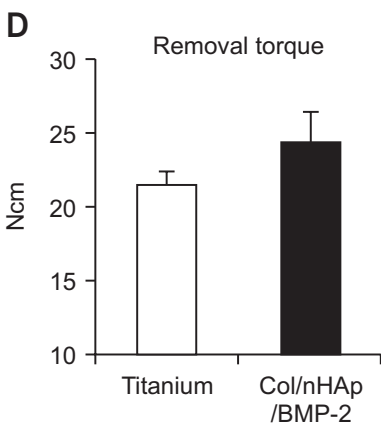

Fig. 6. Implant installation on rabbit tibia. A. Photograph of titanium implant (left) and Col/nHAp/BMP-2-coated implant (right). B-D. Bone implant contact, new bone area, and removal torque. At 4 weeks after implant installation on rabbit tibia, Col/nHAp/BMP-2 exhibited increased new bone area compared with the negative control $(P=0.07)$, whereas bone implant contact and removal torque exhibited no significant difference, although the mean values were higher. (Col: collagen, nHAp: nano-hydroxyapatite, BMP-2: bone morphogenetic protein-2)

KangMi Pang et al: Effects of the combination of bone morphogenetic protein-2 and nano-hydroxyapatite on the osseointegration of dental implants. J Korean Assoc Oral Maxillofac Surg 2021

control $(22.90 \pm 2.33 \mathrm{Ncm})$.

\section{Discussion}

This study aimed to develop an implant surface that would allow the active growth factor BMP-2 to promote osseointegration. We thus investigated the biological response to BMP-2 combined with nHAp on titanium in vitro and in vivo. The results indicated that the coating potently increased osteoblastic differentiation of human BM-MSCs in vitro.

As a BMP-2 carrier, HAp also exhibited promising results. Recent studies with 5 different ceramic additives, that is, $\beta$-tricalcium phosphate and 4 types of HAp (e.g., nHAp, HAp), in the ectopic bone formation model of the rat thigh muscle have reported a higher bone density with $20 \mathrm{~nm}$ HAp than with the other additives ${ }^{17}$. Other studies found that BMP7-transduced MSCs, seeded on nHAp composite, initially accelerated bone regeneration after implantation ${ }^{18}$. Using a rabbit model, Liu et al. ${ }^{19}$ demonstrated that an rhBMP-2-loaded gelatin/nHAp scaffold exhibited good osteogenic capability and could repair a segmental bone defect completely in 12 weeks. These findings were analogous to our results in vitro, which revealed osteoblastic differentiation of stem cells on titanium coated with nHAp and BMP-2. However, hydrophilicity was decreased when adding the nHAp on a collagen surface. Surface wettability was reported to be altered by biomaterial surface roughness, composition, and the presence of nanofeatures, and the effect of nHAp on surface wettability was controversial. These may be affected by the composition of the nHAp, such as the ratio of $\mathrm{Ca} / \mathrm{P}$, and further study would be necessary.
To study the influence of nHAp-BMP-2 on the differentiation of the BM-MSCs, we evaluated the expression of stem cell markers, as well as phenotypic markers of osteoblasts and osteogenic genes. FACS analysis demonstrated a loss of stemness with decreasing expression of CD73, CD90, and CD105. Biochemical assays revealed the highest levels of ALP, GAG, and OPN in Col/nHAp/BMP-2, followed by Col/ nHAp. ALP activity is a phenotypic marker of osteoblasts and is characteristic of differentiation ${ }^{20}$. GAG is the major organic extracellular matrix component, and changes in its composition are frequently seen in physiological and pathological remodeling processes such as bone formation or scarring $^{21}$. The expression of OPN by osteoblasts early in bone development is consistent with the formation of the bone matrix $^{22}$. Thus, higher ALP activity and greater expression of GAG and OPN in BM-MSCs on Col/nHAp/BMP-2 indicated that nHAp with BMP-2 can induce BM-MSCs to osteoblastic differentiation after culture for 14 days. These activities also increased on $\mathrm{Col} / \mathrm{nHAp}$, although to a lesser extent than for $\mathrm{Col} / \mathrm{nHAp} / \mathrm{BMP}-2$. These results demonstrated the osteoinductive capacity of nHAp itself and the synergistic effect of coupling BMP-2 with nHAp.

The RT-PCR results revealed higher BMP-2 expression of cells on $\mathrm{Col} / \mathrm{nHAp}$ and $\mathrm{Col} / \mathrm{nHAp} / \mathrm{BMP}-2$ than on Col. For OPN and osteonectin levels, the early marker of bone development, similar patterns of expression were observed. These results also support the role of nHAp in osteoblast differentiation. A study by Prosecká et al ${ }^{23}$ reported a similar pattern in which a mixture of HAp and collagen could induce the osteogenic differentiation of MSCs. Expressions of osteocalcin and osteoprotegerin were highest in Col/nHAp/ 
BMP-2. Osteocalcin is secreted solely by osteoblasts and is believed to play a role in bone mineralization and calcium ion homeostasis ${ }^{24}$. Osteoprotegerin can reduce the production of osteoclasts by inhibiting the differentiation of osteoclast precursors into osteoclasts, and it also regulates the resorption of osteoclasts in vitro and in vivo ${ }^{25}$. Considering these results and those from previous studies, it is possible that BMP-2 combined with nHAp stimulates the formation and mineralization of the bone due to their chemical or structural characteristics.

The mRNA expression levels of type I collagen were highest in BM-MSCs cultured on Col/nHAp, followed by $\mathrm{Col} /$ nHAp/BMP-2, while the expression level of type III collagen was highest on Col/nHAp/BMP-2. Type I collagen is the main component of the organic part of bone, whereas type III collagen, a major component of the extracellular matrix, is commonly found alongside type I collagen and is produced before the tougher type I collagen is synthesized ${ }^{26,27}$. Some studies have reported that BMP-2 inhibits collagen synthesis. Song et al. ${ }^{28}$ reported that the expression of mRNA for collagen types II and V was significantly down-regulated following treatment with rhBMP-2, while the effect of rhBMP-2 on the expression of mRNA for type I collagen was minimal, and the expression of mRNA for type III collagen was enhanced in a dose-dependent manner by treatment with BMP2. No mechanism has yet been proposed to explain the effects of BMP-2 on collagen synthesis, although data from this study suggest a diverse role for BMP-2 on collagen formation.

The mouse subcutaneous injection model did not show any obvious bone formation in any of the groups at 4 weeks. Other studies using BMP-2 in ectopic bone formation models have reported high bone density on histological assessment, with dense bone, lacunae, ossification centers, osteoblasts, and osteocytes ${ }^{17}$. Although no obvious bone formations were observed either macroscopically or microscopically, the Col/ nHAp/BMP-2 group strongly expressed osteonectin, an early marker of osteoblast differentiation, indicating a potential for bone formation after long periods ${ }^{29}$.

Treating the implant surface with bioactive materials such as HAp and BMP-2 has been studied recently to promote osseointegration. Shi et al. ${ }^{30}$ developed a sustained BMP-2 release system based on rhBMP-2 encapsulated poly-D,Llactide-co-glycolide microspheres-loaded chitosan/collagen scaffolds and demonstrated improved osseointegration ability. Chatzinikolaidou et al. ${ }^{31}$ demonstrated the feasibility of enhancing peri-implant osseointegration and gap bridging by using immobilized BMP-2 on implant surfaces. Implants soaked in $2 \mathrm{~mL}$ of sterile water containing $1.0 \mathrm{mg}$ BMP-2 with polylactic acid as the carrier exhibited both qualitative and quantitative improvements in osseointegration at the implant-bone interface ${ }^{32}$.

However, the results of our rabbit tibia implant model suggested that implants coated with BMP-2 and nHAp failed to produce any significant statistical differences, although the mean values for BIC, new bone area, and removal torque were higher. This could be due either to the BMP-2 release from the nHAp/BMP-2 coated implant or the role of BMP in bone formation.

In the release kinetics of BMP-2, most of the coated BMP2 was released within 5 days for the Col/nHAp/BMP-2 group. Although BMP-2 incorporated with nHAp exhibited a slower release pattern than BMP-2 alone, recent studies have reported more sustained release patterns ${ }^{33}$. Hence, the similar results observed between the collagen implant and BMP-2 combined with nHAp-coated implants would be explained by our relatively rapid release of BMP-2 compared to that seen in other studies. Therefore, a study with a 2-week observation period after placement of the BMP-2 implant would be necessary.

There remains some controversy regarding the efficacy of BMP in bone formation, as several factors are known to affect the osteoinductive ability of BMP-2. Recently, it has been reported that BMP-2 promotes the adipogenic differentiation of periodontal ligament stem cells both in vitro and in vivo, in association with a reduced amount of cementum and periodontal ligament formation ${ }^{28}$. Other studies have also reported that BMP-2 significantly enhances the adipogenic as well as osteogenic potential of alveolar bone-derived stromal cells in a dose- and time-dependent manner ${ }^{5}$. The inhibitory effect of rhBMP-2 on collagen synthesis is related to the down-regulation of the minor, rather than the major, components of collagen ${ }^{28}$. In addition, BMP-2 stimulates both the formation and resorption of bone at high doses ${ }^{34}$. Although the presently used loading dose of BMP-2 is very low according to current clinical standards, it still might be excessive in this bony environment. Or on the contrary, our results could be due to too low a concentration of BMP-2. During the placement process of the implant, BMP-2 may be reduced greatly, and further study to rigidly fix the BMP-2 on the implant would be necessary. 


\section{Conclusion}

BMP-2 combined with nHAp induced mesenchymal stem cell differentiation and osteoblastic differentiation in vitro and in vivo with the mouse pocket injection model, although no significant differences were observed in the rabbit tibia implant model. Therefore, our study suggests that BMP-2 combined with a nHAp carrier has the potential to function as an osseointegration activator on the surfaces of dental implants.

\section{ORCID}

KangMi Pang, https://orcid.org/0000-0001-9148-9080

Young-Kwon Seo, https://orcid.org/0000-0001-7533-9605

Jong-Ho Lee, https://orcid.org/0000-0002-8843-545X

\section{Authors' Contributions}

K.M.P. participated in animal experiment and wrote the manuscript. Y.K.S. participated in the study design and performed in vitro experiments. J.H.L. participated in the study design and coordination and helped to draft the manuscript. All authors read and approved the final manuscript.

\section{Ethics Approval and Consent to Participate}

The animal experiments were approved by the Animal Care and Use Committee, Seoul National University (SNU100122-8).

\section{Conflict of Interest}

No potential conflict of interest relevant to this article was reported.

\section{References}

1. Coelho PG, Granjeiro JM, Romanos GE, Suzuki M, Silva NR, Cardaropoli G, et al. Basic research methods and current trends of dental implant surfaces. J Biomed Mater Res B Appl Biomater 2009;88:579-96. https://doi.org/10.1002/jbm.b.31264

2. Dohan Ehrenfest DM, Coelho PG, Kang BS, Sul YT, Albrektsson T. Classification of osseointegrated implant surfaces: materials, chemistry and topography. Trends Biotechnol 2010;28:198-206. https:// doi.org/10.1016/j.tibtech.2009.12.003

3. Mendonça G, Mendonça DB, Aragão FJ, Cooper LF. Advancing dental implant surface technology--from micron- to nanotopography. Biomaterials 2008;29:3822-35. https://doi.org/10.1016/ j.biomaterials.2008.05.012
4. Jovanovic SA, Hunt DR, Bernard GW, Spiekermann H, Nishimura R, Wozney JM, et al. Long-term functional loading of dental implants in rhBMP-2 induced bone. A histologic study in the canine ridge augmentation model. Clin Oral Implants Res 2003;14:793803. https://doi.org/10.1046/j.0905-7161.2003.clr140617.x

5. Park JC, Kim JC, Kim BK, Cho KS, Im GI, Kim BS, et al. Doseand time-dependent effects of recombinant human bone morphogenetic protein- 2 on the osteogenic and adipogenic potentials of alveolar bone-derived stromal cells. J Periodontal Res 2012;47:64554. https://doi.org/10.1111/j.1600-0765.2012.01477.x

6. Lee JH, Yu CH, Yang JJ, Baek HR, Lee KM, Koo TY, et al. Comparative study of fusion rate induced by different dosages of Escherichia coli-derived recombinant human bone morphogenetic protein-2 using hydroxyapatite carrier. Spine J 2012;12:239-48. https://doi.org/10.1016/j.spinee.2012.01.013

7. Fujimura K, Bessho K, Kusumoto K, Ogawa Y, Iizuka T. Experimental studies on bone inducing activity of composites of atelopeptide type I collagen as a carrier for ectopic osteoinduction by rhBMP-2. Biochem Biophys Res Commun 1995;208:316-22. https://doi.org/10.1006/bbrc.1995.1340

8. Garrett MP, Kakarla UK, Porter RW, Sonntag VK. Formation of painful seroma and edema after the use of recombinant human bone morphogenetic protein-2 in posterolateral lumbar spine fusions. Neurosurgery 2010;66:1044-9; discussion 1049. https://doi. org/10.1227/01.NEU.0000369517.21018.F2

9. Kokorina NA, Lewis JS Jr, Zakharkin SO, Krebsbach PH, Nussenbaum B. rhBMP-2 has adverse effects on human oral carcinoma cell lines in vivo. Laryngoscope 2012;122:95-102. https://doi. org/10.1002/lary.22345

10. Koyama N, Okubo Y, Nakao K, Osawa K, Bessho K. Experimental study of osteoinduction using a new material as a carrier for bone morphogenetic protein-2. Br J Oral Maxillofac Surg 2011;49:3148. https://doi.org/10.1016/j.bjoms.2010.05.010

11. Mindea SA, Shih P, Song JK. Recombinant human bone morphogenetic protein-2-induced radiculitis in elective minimally invasive transforaminal lumbar interbody fusions: a series review. Spine (Phila Pa 1976) 2009;34:1480-4; discussion 1485. https://doi. org/10.1097/BRS.0b013e3181a396a1

12. Perri B, Cooper M, Lauryssen C, Anand N. Adverse swelling associated with use of rh-BMP-2 in anterior cervical discectomy and fusion: a case study. Spine J 2007;7:235-9. https://doi.org/10.1016/ j.spinee.2006.04.010

13. Smoljanovic T, Pecina M. Re: Burkus J K, Sandhu H S, Gornet M F. Influence of rhBMP-2 on the healing patterns associated with allograft interbody constructs in comparison with autograft. Spine 2006;31:775-81. Spine (Phila Pa 1976) 2008;33:226. https://doi. org/10.1097/BRS.0b013e3181606894

14. Wong DA, Kumar A, Jatana S, Ghiselli G, Wong K. Neurologic impairment from ectopic bone in the lumbar canal: a potential complication of off-label PLIF/TLIF use of bone morphogenetic protein-2 (BMP-2). Spine J 2008;8:1011-8. https://doi.org/10.1016/ j.spinee.2007.06.014

15. Wen B, Karl M, Pendrys D, Shafer D, Freilich M, Kuhn L. An evaluation of BMP-2 delivery from scaffolds with miniaturized dental implants in a novel rat mandible model. J Biomed Mater Res B Appl Biomater 2011;97:315-26. https://doi.org/10.1002/jbm. b. 31817

16. Zhang W, Tsurushima H, Oyane A, Yazaki Y, Sogo Y, Ito A, et al. BMP-2 gene-fibronectin-apatite composite layer enhances bone formation. J Biomed Sci 2011;18:62. https://doi.org/10.1186/14230127-18-62

17. Hulsart-Billström $\mathrm{G}, \mathrm{Hu} \mathrm{Q}$, Bergman $\mathrm{K}$, Jonsson $\mathrm{KB}$, Åberg J, Tang $\mathrm{R}$, et al. Calcium phosphates compounds in conjunction with hydrogel as carrier for BMP-2: a study on ectopic bone formation in rats. Acta Biomater 2011;7:3042-9. https://doi.org/10.1016/ j.actbio.2011.04.021

18. Li J, Li Y, Ma S, Gao Y, Zuo Y, Hu J. Enhancement of bone forma- 
tion by BMP-7 transduced MSCs on biomimetic nano-hydroxyapatite/polyamide composite scaffolds in repair of mandibular defects. J Biomed Mater Res A 2010;95:973-81. https://doi.org/10.1002/ jbm.a.32926

19. Liu Y, Lu Y, Tian X, Cui G, Zhao Y, Yang Q, et al. Segmental bone regeneration using an rhBMP-2-loaded gelatin/nanohydroxyapatite/fibrin scaffold in a rabbit model. Biomaterials 2009;30:627685. https://doi.org/10.1016/j.biomaterials.2009.08.003

20. Gomez B Jr, Ardakani S, Ju J, Jenkins D, Cerelli MJ, Daniloff GY, et al. Monoclonal antibody assay for measuring bone-specific alkaline phosphatase activity in serum. Clin Chem 1995;41:1560-6.

21. Salbach J, Rachner TD, Rauner M, Hempel U, Anderegg U, Franz $\mathrm{S}$, et al. Regenerative potential of glycosaminoglycans for skin and bone. J Mol Med (Berl) 2012;90:625-35. https://doi.org/10.1007/ s00109-011-0843-2

22. Chen J, Singh K, Mukherjee BB, Sodek J. Developmental expression of osteopontin (OPN) mRNA in rat tissues: evidence for a role for OPN in bone formation and resorption. Matrix 1993;13:113-23. https://doi.org/10.1016/s0934-8832(11)80070-3

23. Prosecká E, Rampichová M, Vojtová L, Tvrdík D, Melčáková $S$, Juhasová $\mathrm{J}$, et al. Optimized conditions for mesenchymal stem cells to differentiate into osteoblasts on a collagen/hydroxyapatite matrix. J Biomed Mater Res A 2011;99:307-15. https://doi. org/10.1002/jbm.a.33189

24. Lee NK, Sowa H, Hinoi E, Ferron M, Ahn JD, Confavreux C, et al. Endocrine regulation of energy metabolism by the skeleton. Cell 2007;130:456-69. https://doi.org/10.1016/j.cell.2007.05.047

25. Blázquez-Medela AM, López-Novoa JM, Martínez-Salgado C. Osteoprotegerin and diabetes-associated pathologies. Curr Mol Med 2011;11:401-16. https://doi.org/10.2174/156652411795976565

26. Marks SC Jr, Odgren PR. Chapter 1 - structure and development of the skeleton. In: Bilezikian JP, Raisz LG, Rodan GA, eds. Principles of bone biology. 2nd ed. New York (NY): Academic Press; 2002:3-15.

27. Liu X, Wu H, Byrne M, Krane S, Jaenisch R. Type III collagen is crucial for collagen I fibrillogenesis and for normal cardiovascular development. Proc Natl Acad Sci U S A 1997;94:1852-6. https:// doi.org/10.1073/pnas.94.5.1852

28. Song DS, Park JC, Jung IH, Choi SH, Cho KS, Kim CK, et al. Enhanced adipogenic differentiation and reduced collagen synthesis induced by human periodontal ligament stem cells might underlie the negative effect of recombinant human bone morphogenetic protein-2 on periodontal regeneration. J Periodontal Res 2011;46:193203. https://doi.org/10.1111/j.1600-0765.2010.01328.x

29. Nakashima K, Zhou X, Kunkel G, Zhang Z, Deng JM, Behringer $\mathrm{RR}$, et al. The novel zinc finger-containing transcription factor osterix is required for osteoblast differentiation and bone formation. Cell 2002;108:17-29. https://doi.org/10.1016/s00928674(01)00622-5

30. Shi S, Cheng X, Wang J, Zhang W, Peng L, Zhang Y. RhBMP-2 microspheres-loaded chitosan/collagen scaffold enhanced osseointegration: an experiment in dog. J Biomater Appl 2009;23:331-46. https://doi.org/10.1177/0885328208090013

31. Chatzinikolaidou M, Lichtinger TK, Müller RT, Jennissen HP. Peri-implant reactivity and osteoinductive potential of immobilized rhBMP-2 on titanium carriers. Acta Biomater 2010;6:4405-21. https://doi.org/10.1016/j.actbio.2010.06.009

32. Lan J, Wang ZF, Shi B, Xia HB, Cheng XR. The influence of recombinant human BMP-2 on bone-implant osseointegration: biomechanical testing and histomorphometric analysis. Int $\mathrm{J}$ Oral Maxillofac Surg 2007;36:345-9. https://doi.org/10.1016/ j.ijom.2006.10.019

33. Bae IH, Yun KD, Kim HS, Jeong BC, Lim HP, Park SW, et al. Anodic oxidized nanotubular titanium implants enhance bone morphogenetic protein-2 delivery. J Biomed Mater Res B Appl Biomater 2010;93:484-91. https://doi.org/10.1002/jbm.b.31606

34. Shi J, Dong LL, He F, Zhao S, Yang GL. Osteoblast responses to thin nanohydroxyapatite coated on roughened titanium surfaces deposited by an electrochemical process. Oral Surg Oral Med Oral Pathol Oral Radiol 2013;116:e311-6. https://doi.org/10.1016/ j.00oo.2012.02.021

How to cite this article: Pang K, Seo YK, Lee JH. Effects of the combination of bone morphogenetic protein-2 and nanohydroxyapatite on the osseointegration of dental implants. J Korean Assoc Oral Maxillofac Surg 2021;47:454-464. https://doi. org/10.5125/jkaoms.2021.47.6.454 